\section{Comparative study of the subjective and objective grading of ptosis surgery outcomes}

\author{
Abstract \\ Aims To assess the results of blepharoptosis \\ surgery in our hospital by subjective and \\ objective grading of the outcome and \\ comparing them to determine their degree of \\ corelation. \\ Methods Retrospective interventional case \\ series report supplemented by postal \\ questionnaires and telephonic patient \\ surveys. The study included 107 eyelids \\ of 78 patients. Using a simple grading system, \\ surgical outcome was objectively graded \\ as good, suboptimal, or poor. Outcome \\ was also defined according to the patients' \\ perspective as good, suboptimal, or poor. \\ Level of agreement between the subjective \\ and objective grading of the outcome \\ was measured using a weighted kappa \\ analysis. \\ Results The objective results were classed \\ as good-68/107 (63.5\%), suboptimal-18 \\ eyelids (16.8\%), and poor-21 eyelids \\ (19.6\%). The subjective results were \\ obtainable in 91 eyelids and were good- \\ $54 / 91$ (59.3\%), suboptimal-8/91 (8.7\%), and \\ poor-29/91 (32\%). A mismatch between \\ objective and subjective outcomes was \\ seen in 16 eyelids. We saw a statistically \\ significant corelation between the objective \\ grading and the patients' perspective \\ $(P<0.001)$. \\ Conclusion Our overall ptosis surgery \\ results are comparable with rates \\ previously reported. The subjective \\ and objective outcomes of ptosis \\ surgery may sometimes vary, but \\ nevertheless exhibit substantive agreement \\ when measured by this simple grading \\ system. \\ Eye (2007) 21, 639-642. doi:10.1038/sj.eye.6702296; \\ published online 24 February 2006
}

K Taherian', PL Atkinson', M Shekarchian' and AJ Scally ${ }^{2}$

Keywords: ptosis; blepharoptosis; ptosis surgery outcome

\section{Introduction}

Blepharoptosis is a common type of eyelid malposition $^{1}$ found in all age groups and has multiple causes. ${ }^{2}$ It can cause amblyopia ${ }^{3}$ and affect visual function, but is most often a cosmetic problem. Most, but not all, types of blepharoptosis are treated surgically. ${ }^{4,5}$

The aim of this study was to assess the results of blepharoptosis surgery as a whole in our hospital by subjective and objective grading of the outcome and compare them to determine their degree of corelation.

\section{Materials and methods}

This study is a retrospective interventional case series report supplemented by postal

questionnaires and telephonic patient surveys. The medical records of 78 consecutive patients (107 eyes) who had undergone blepharoptosis surgery at our hospital between March 1995 and February 2004 were reviewed.

The following variables were extracted from the patients' records: age, gender, date of presentation, length of history, family history of ptosis, degree of ptosis, laterality, amount of ptosis (mm), previous ptosis surgery, type of ptosis, levator function, date of surgery, surgeons' grade, type of anaesthesia, type of surgical approach (anterior/posterior), ${ }^{5}$ type of surgical procedure, postoperative problems if any, need for repeat surgery, and follow-up duration. In unilateral cases, the amount of ptosis was calculated as the difference (mm) between heights of the palpaebral apertures. Mild ptosis was defined as $2 \mathrm{~mm}$ or less,
${ }^{1}$ Department of Ophthalmology, Bradford Royal Infirmary, Bradford BD9 6RJ, UK

${ }^{2}$ School of Health Studies, University of Bradford, Bradford BD5 OBB, UK

Correspondence: K Taherian, Ophthalmology, St James' University Hospital, Beckett Street, Leeds, West Yorkshire LS9 7TF, UK

Tel: + 44798061 4907; Fax: +44 1132648775 . E-mail: kasra_taherian@ yahoo.co.uk

Received: 2 August 2005 Accepted in revised form: 15 January 2006 Published online: 24 February 2006

The study was carried out at the Department of Ophthalmology Bradford Royal Infirmary, Bradford BD9 6RJ, UK 
moderate ptosis was defined as $2-4 \mathrm{~mm}$, and severe ptosis was defined as $4 \mathrm{~mm}$ or more lower than the desired upper eyelid level. ${ }^{1}$ In bilateral cases, the ptosis was classified as severe if the height of the palpaebral fissures was less than or equal to $4 \mathrm{~mm}$, as moderate if the palpaebral apertures were between 4 and $6 \mathrm{~mm}$, and mild if the palpaebral apertures were $6 \mathrm{~mm}$ or more. ${ }^{1}$ Levator function was measured as the maximum lid excursion from maximal downgaze to upgaze, with frontalis function abolished by pressing on the eyebrow. This was recorded as poor if less than $4 \mathrm{~mm}$, moderate if between 4 and $8 \mathrm{~mm}$, and good if more than $8 \mathrm{~mm} .{ }^{1}$ Outcome was objectively defined as good if the lids were within $1 \mathrm{~mm}$ height with an acceptable skin crease and contour with no corneal exposure and as poor if reoperation was required. ${ }^{1}$ Another group was identified as suboptimal outcome - where there was more than $1 \mathrm{~mm}$ difference in lid height, and/or an asymmetric skin crease and/or contour but who did not undergo further operations. Outcome was also defined according to the patients' perspective as good, suboptimal or poor. The patients perspective was obtained by a combination of recordings from case notes, postal questionnaires, and telephone surveys.

\section{Description of the statistical analysis}

The level of agreement between the subjective and objective grading of the outcome was measured using a weighted kappa analysis ${ }^{6,7}$ ignoring cases where the subjective measure was missing (17 cases when all eyes were analysed; 12 cases when a random eye was selected from bilateral cases). Both subjective and objective measures were on a three-point scale $(1=$ poor; 2 =moderate; 3 =good). Complete agreement was given a weighting factor of 1 , disagreement by one point was weighted 0.5 , and disagreement by two points was weighted zero.

As there was a reasonable likelihood that the correlation between the subjective and objective measures would be stronger for within patient measurements, that is for the bilateral cases, initially one eye was selected at random for analysis from the bilateral cases. Although this reduced the sample size available for analysis, it also reduced the potential for bias. The kappa statistic was recalculated for all eyelids, to enable a subjective comparison with the kappa calculated using a single eyelid per case and to improve the precision of the estimate.

\section{Results}

The study included 107 eyes of 78 patients. The age of the patients ranged between 2 and 95 years (median 43 years). There were 38 male and 40 female patients with a male: female ratio of $0.95: 1$. Only nine cases had a positive family history of ptosis. The degree of ptosis was mild in $32(30 \%)$, moderate in $45(42 \%)$ and severe in 30 $(28 \%)$ eyes. The length of history ranged between 2 and 1032 months (mean 120.67). The amount of ptosis ranged between 2 and $8 \mathrm{~mm}$, but had not been recorded in nine eyelids. A total of $12(11 \%)$ eyelids had had previous ptosis surgery.

The right side was the only operated eyelid in 25 $(23.3 \%)$ of cases, and the left side in $18(16.7 \%)$ and 32 patients (60\% of the eyelids) had bilateral surgery. Jaw winking was documented to be absent in $92(85.5 \%)$ of eyelids and not recorded in $15(14.5 \%)$. Levator function was good in $54(50.5 \%)$ eyelids, moderate in $30(28 \%)$, poor in $13(12.2 \%)$, and was not recorded for $10(9.3 \%)$ eyes. The operating surgeon was of consultant grade in $94(88 \%)$ and the rest were operated by trainees.

Out of the 91 eyelids for which the subjective outcome was obtainable, the information was obtained from the case notes for $60(65.9 \%)$ eyelids, by means of postal questionnaires for 12 (13.2\%) eyelids, and by telephone surveys for the remaining 19 (20.9\%) eyelids (Table 1).

General anaesthesia was used in $50(47 \%)$ cases and 57 (53\%) had local anaesthesia. An anterior surgical approach was used in 103 (96.5\%) eyelids and a posterior approach in four cases (3.5\%) (Table 2).

Postoperative problems were noted in $38(35 \%)$ eyelids, which were overcorrection in nine eyelids (8.5\%), undercorrection in $24(22.4 \%)$, significant lagophthalmos in one $(0.9 \%)$, irregular contour in one $(0.9 \%)$, and low skin crease in one eyelid $(0.9 \%)$.

Table 1 Type of Ptosis

\begin{tabular}{lc}
\hline Type of Ptosis & Number of eyes (107) \\
\hline Congenital & $42(39.3 \%)$ \\
Aponeurotic & $41(38.2 \%)$ \\
Neurogenic & $4(3.7 \%)$ \\
Myogenic & $10(9.3 \%)$ \\
Post-traumatic & $5(4.7 \%)$ \\
Mechanical & $1(1 \%)$ \\
Not recorded & $3(2.8 \%)$ \\
\hline
\end{tabular}

Table 2 Type of surgical procedure

\begin{tabular}{lc}
\hline Type of surgical procedure & Number of eyes (107) \\
\hline Levator resection & $36 / 107(33.6 \%)$ \\
Aponeurotic repair & $40 / 107(37.3 \%)$ \\
Brow suspension with fascia lata & $12 / 107(11.2 \%)$ \\
Brow suspension with silicone sling & $14 / 107(13 \%)$ \\
Fasanella servat procedure & $4 / 107(3.5 \%)$ \\
Tarsal plate suspension suture & $1 / 107(0.9 \%)$ \\
\hline
\end{tabular}


Table 3 Classification of the outcome of surgery according to objective criteria

\begin{tabular}{lcll}
\hline $\begin{array}{l}\text { Objective } \\
\text { outcome }\end{array}$ & Good & Sub-optimal & Poor \\
\hline $\begin{array}{l}\text { No. of } \\
\text { eyes (107) }\end{array}$ & $68 / 107(63.5 \%)$ & $18 / 107(16.8 \%)$ & $21 / 107(19.6 \%)$ \\
\hline
\end{tabular}

Table 4 Classification of the outcome of surgery according to subjective criteria

\begin{tabular}{lccl}
\hline $\begin{array}{l}\text { Subjective } \\
\text { outcome }\end{array}$ & Good & Sub-optimal & Poor \\
\hline $\begin{array}{l}\text { No. of } \\
\text { eyes (91) }\end{array}$ & $54 / 91(59.3 \%)$ & $8 / 91(8.7 \%)$ & $29 / 91(32 \%)$ \\
\hline
\end{tabular}

Table 5 Cross-tabulations of the objective and subjective scores (Single eye per patient)

\begin{tabular}{lccc}
\hline Subjective measure & \multicolumn{3}{c}{ Objective measure } \\
\cline { 2 - 4 } & 1 (poor) & 2 (moderate) & 3 (good) \\
\hline 1 (poor) & 15 & 3 & 2 \\
2 (moderate) & 0 & 3 & 3 \\
3 (good) & 0 & 3 & 37 \\
missing & 0 & 4 & 8 \\
\hline
\end{tabular}

The duration of postoperative follow-up ranged between 1 and 96 months (mean 20.1) (Table 3).

Subjective data for 91/107 (85\%) eyelids were obtainable and hence the rest of the eyelids were excluded from the assessment of the correlation between the objective and subjective outcome (Table 4).

\section{Correlation between the objective and subjective outcome}

Out of the 91 (82.4\%) eyelids for which both objective and subjective outcomes were known, a mismatch between the objective and subjective outcomes was seen in 16 eyelids. Of these, for four eyelids they reported a better subjective outcome than the objective grade, and for 12 eyelids they reported a lower subjective outcome than the objective grade (Tables $5-7$ ).

There is very little difference in the observed agreement and kappa values when comparing the analysis of all eyelids with that using a single eyelid per case. Both analyses produced a statistically significant kappa value $(P<0.001$ in both cases) that represented substantial agreement between the subjective and
Table 6 Cross-tabulations of the objective and subjective scores (All eyes)

\begin{tabular}{lccc}
\hline Subjective measure & \multicolumn{3}{c}{ Objective measure } \\
\cline { 2 - 4 } & 1 (poor) & 2 (moderate) & 3 (good) \\
\hline 1 (poor) & 21 & 5 & 3 \\
2 (moderate) & 0 & 4 & 4 \\
3 (good) & 0 & 4 & 50 \\
missing & 0 & 5 & 12 \\
\hline
\end{tabular}

Table 7 Weighted Kappa statistics for single eye per patient and for all eyelids

\begin{tabular}{lccccc}
\hline & Agreement & $\begin{array}{c}\text { Expected } \\
\text { agreement }\end{array}$ & Kappa & Std. err. & P-value \\
\hline $\begin{array}{l}\text { Single eye per } \\
\text { patient }\end{array}$ & 90.15 & 56.82 & 0.7719 & 0.1087 & $<0.001$ \\
\begin{tabular}{l} 
All eyes \\
\hline
\end{tabular} & 89.56 & 56.06 & 0.7624 & 0.0923 & $<0.001$ \\
\hline
\end{tabular}

objective outcomes, as per the subjective interpretation suggested by Landis and Koch. ${ }^{6}$

\section{Discussion}

The commonest objective definition of success in ptosis surgery is lid levels within $1 \mathrm{~mm}$ of each other postoperatively. ${ }^{8}$ Although this definition is simple to define and measure, it does not consider the patients' perspective of the outcome, which in our opinion is the ultimate measure of success (at least in adults). To our knowledge, this is the first study to compare the subjective and objective grading of ptosis surgery outcomes and statistically measure their degree of corelation.

Although the predictability of the outcome of ptosis surgery is enhanced by using local anaesthesia, ${ }^{9}$ we had to operate nearly half of our patients under General anaesthesia because of the relative proportion of our paediatric patients as well as patient choice. Yet our objective surgical outcome compared favourably with the other reported success rates. ${ }^{1,9,10}$

The higher proportion of surgical outcomes graded subjectively as poor when compared with the objective grading may be attributable to the fact that whereas the objective assessment took place within 3 months of surgery (typically 6 weeks), ${ }^{11}$ some of the subjective data were collected by means of postal questionnaires or telephone interviews long after the date of surgery and hence would include ptosis recurrences.

The overall results of ptosis surgery in our setting appear satisfactory. The subjective and objective 
outcomes of ptosis surgery may sometimes vary; nevertheless, our results suggest a substantive and statistically significant corelation between objective criteria used here to assess the outcome and the patients' perspective. However, larger, prospective, and preferably multicentric studies would be desirable to prove this more conclusively.

\section{References}

1 Lee V, Konrad H, Bunce C, Nelson C, Collin JRO. Aetiology and surgical treatment of childhood blepharoptosis. $\mathrm{Br} \mathrm{J}$ Ophthalmol 2002; 86: 1282-1286.

2 Freuh HR. The mechanistic classification of ptosis. Ophthalmology 1980; 87: 1019-1021.

3 Anderson RL, Baumgarter SA. Amblyopia in ptosis. Arch Ophthalmol 1980; 98: 1068-1069.
4 Collin JRO. Complications of ptosis surgery and their management. J R Soc Med 1979; 72: 25-26.

5 Collin JRO. A ptosis repair of aponeurotic defects by the posterior approach. Br J Ophthalmol 1979; 63: 586-590.

6 Landis JR, Koch GG. The measurement of observer agreement for categorical data. Biometrics 1977; 33: 159-174.

7 StataCorp. Stata Statistical Software: Release 8.2. Stata Corporation: College Station, TX, 2005.

8 Cates CA, Tyers AG. Outcomes of anterior levator resection in congenital blepharoptosis. Eye 2001; 15: 770-773.

9 Linberg JV, Vasquez RJ, Chao GM. Aponeurotic ptosis repair under local anaesthesia. Ophthalmology 1988; 95: 1046-1052.

10 Liu D. Blepharoptosis correction with frontalis suspension using a supramid sling: duration of effect. Am J Ophthalmol 1999; 128(6): 772-773.

11 Tucker SM, Verhulst SJ. Stabilisation of eyelid height after aponeurosis ptosis repair. Ophthalmology 1999; 106: 517-522. 